Voix et Images

\title{
La dialectique de l'ancien et du nouveau chez Marie-Claire Blais, Jacques Ferron et Réjean Ducharme
}

\section{Gilles Marcotte}

Volume 6, numéro 1, automne 1980

Gilles Marcotte

URI : https://id.erudit.org/iderudit/200250ar

DOI : https://doi.org/10.7202/200250ar

Aller au sommaire du numéro

Éditeur(s)

Les Presses de l'Université du Québec

ISSN

0318-9201 (imprimé)

1705-933X (numérique)

Découvrir la revue

Citer cet article

Marcotte, G. (1980). La dialectique de l'ancien et du nouveau chez Marie-Claire Blais, Jacques Ferron et Réjean Ducharme. Voix et Images, 6(1), 63-73.

https://doi.org/10.7202/200250ar d'utilisation que vous pouvez consulter en ligne. 


\title{
La dialectique de l'ancien et du nouveau chez Marie-Claire Blais, Jacques Ferron et Réjean Ducharme
}

\author{
par Gilles Marcotte
}

Pourquoi, dans Le ciel de Québec, Jacques Ferron donne-t-il un rôle si important, et si sympathique, à Monseigneur Camille Roy? À lui, les premières lignes du roman: "Monseigneur Camille, de la lignée humaniste des prélats québecquois, homme bon, discret et de bonne compagnie.... Un peu d'ironie, peut-être, dans cette description, mais une ironie bonhomme, tempérée par une authentique considération. Monseigneur $\mathrm{Ca}$ mille Roy est un personnage bien connu - comme, d'ailleurs, la plupart des personnages du Ciel de Québec. Nous avons lu les multiples éditions de son Manuel d'histoire de la littérature canadienne de langue française et ses nombreuses études, qui ne pèchent certes pas par excès de libéralisme. Nous avons même accoutumé de voir en lui le parfait exemple d'une critique bornée, hostile à tout modernisme, aisément effarouchée par le moindre souffle de liberté; une critique, en somme, tournée vers le passé, véhiculant les valeurs du passé. N'est-il pas étonnant qu'un écrivain aussi libre, aussi moderne, que Jacques Ferron, fasse honneur. à un tel personnage, alors que dans le même roman il traitera assez mal, merci, un SaintDenys-Garneau, voire un Paul-Émile Borduas, qui semblent beaucoup plus proche des préoccupations actuelles du Canada français?

Deuxième question: qui est, que représente Grand-Mère Antoinette, dans le roman de Marie-Claire Blais, Une saison dans la vie d'Emmanuel? La tradition, sans doute, ou la «pérennité », comme le dit Lucien Goldmann, qui la décrit comme « une grand-mère campagnarde, immortelle, optimiste, simple, à dimensions presque mythiques" 1 . Un autre critique, Madeleine Greffard, en parle également comme d'un "pôle positif" dans le roman: "Elle aime la vie; sa rudesse pour ses petits-enfants ne va pas sans un grand amour. Elle forme, avec Jean-le-maigre, un pôle positif dans ce monde de silence et de démission. Elle vit, elle aime, bien qu'elle se soumette à la religion conventionnelle du curé, qu'elle en accepte les tabous et les défenses. ${ }^{2}$ Le «bien que» dont Madeleine Greffard assortit la dernière phrase signale cependant une difficulté dans l'interprétation du personnage. Si le roman de Marie-Claire Blais est, comme dit le même critique, "une véritable liquidation de tous les vieux mythes 
canadiens-français ${ }^{3}$, comment expliquer qu'un personnage aussi nettement identifié à la tradition, au passé, avec ses «tabous", ses «défenses", y représente également la force de vie? La source de la vraie vie serait-elle donc malgré tout dans le passé, comme le prônaient Monseigneur Camille Roy et tant d'autres vieux tousseux de notre littérature?

La même question peut être posée à propos de Réjean Ducharme, qui s'en prend avec une vigueur extrême à l'idée même de progrès, et dénonce par exemple la liberté sexuelle, le syndicalisme. On croirait entendre un Rédemptoriste de haute époque lorsqu'il proclame: «Marie de l'Incarnation ne s'est pas laissé berner, elle. Elle est restée belle, blanche, pure et sereine, jusqu'au dernier jour. (...) Je n'aime pas la prostitution désintellectuellement légalisée, financée et organisée. Je n'aime pas le décolleté, la blouse transparente, le dessin de la culotte au travers de la jupe, le rouge à lèvres, le genou à l'air. " ${ }^{4}$ Quant aux syndicalistes, ils sont présentés comme une "bande de gueuleurs, de quêteurs / De baveurs de slogans, de chieurs de pancartes ${ }^{5}$. Oui, certes, je vous entends, la violence du ton, l'excès de telles proclamations nous conduisent parfois aux frontières de la parodie; mais aux frontières seulement, là où le sens tremble, se brouille, se multiplie. II n'en reste pas moins qu'il faut accorder une certaine signification au choix, par Ducharme, de cibles qui représentent généralement, dans l'opinion dite éclairée, des motifs de progrès, de libération.

Ces quelques observations suggèrent, à tout le moins, que les rapports entre l'ancien et le nouveau sont, dans les œuvres de Blais, Ferron et Ducharme, assez complexes, et qu'ils ne sauraient être réduits au schéma naïf suivant lequel le nouveau, en succédant à l'ancien, l'oblitère. Voici donc trois œuvres qui ont, dans notre littérature, une actualité certaine; mais qui, en même temps, entretiennent de singuliers rapports avec les thèmes anciens que notre roman, d'après la critique, avait mis au rancart: la tradition, le refus de la chair et du progrès social, et cetera. Dans un article récent, Jack Warwick s'est même demandé si l'on ne pouvait déceler au Québec un «retour aux mythes de la terre"; et il a répondu, après avoir interrogé quelques romans et poèmes: «...Dans la spécificité des mythes de la terre il n'y a, dans le passage de la littérature dite canadiennefrançaise à la littérature québécoise (i.e. de l'ancienne à la nouvelle), aucune solution de continuité. " 6 Au niveau d'abstraction et de généralité où il considère les thèmes, c'est-à-dire en n'en conservant que la pure essence symbolique, Warwick a raison de parler de continuité. Rien n'empêche de dire, en effet, que l'attachement à la terre exprimé dans tel roman d'André Major ou d'Andrée Maillet, tel poème de Gatien Lapointe ou de Gilles Vigneault, n'est pas fondamentalement différent de celui qu'on lit dans Menaud maitre-draveur ou Maria Chapdelaine. Quand on cherche bien, on découvre toujours, dans les œuvres les plus diverses, des signes de cette continuité, de cette permanence-là. Ce que j'éssaie de mettre au jour, chez Ferron, Blais et Ducharme, est d'un autre ordre. II y a ici référence à l'ancien, à l'ancien comme tel, voire - comme je le montrerai tout 
à l'heure, et comme il apparaît déjà par l'exemple de Monseigneur Camille Roy - au texte ancien. Non pas rencontre, convergence avec les œuvres anciennes dans le même, qu'il soit d'essence symbolique ou autre; mais distance maintenue, affichée, et en même temps convocation par le nouveau de l'ancien, pour qu'il entre avec le nouveau dans un processus de transformation, de change. En d'autres termes, l'ancien n'est pas ici ce qui dure, ce qui se maintient malgré les aléas des transformations sociales et esthétiques; il n'est pas non plus un refuge dans lequel des consciences affolées par le tohu-bohu de la vie moderne iraient chercher un peu de paix et de sécurité; il est l'antithèse nécessaire, dans un mouvement dialectique. Marthe Robert, dans son analyse du donquichottisme, a bien montré la différence qui existe entre un tel mouvement et celui de la simple satire, qui prétend se débarrasser de l'ancien en le faisant lire comme révolu. "Cette idée, dit-elle, selon laquelle une déclaration de péremption suffit à éteindre l'action des choses anciennes, est très exactement l'une des graves illusions que le donquichottisme cherche à détruire, par des moyens qui ne sont ni le persiflage ni la dérision, mais simultanérnent la piété et l'ironie, le respect et l'humour, l'admiration et la critique, l'attendrissement et la rigueur; qui substituent donc au ou bien catégorique de la satire un et déchirant, maintenu jusqu'aux limites de l'absurde. ${ }^{7}$ Ainsi, l'ancien et le nouveau entrent dans une relation de coordination - le «et déchirant»- qui se joue à la fois de l'identité et de la contradiction, de la subordination et de l'égalité, de la succession et de la simultanéité.

De cette relation, les figures de Grand-Mère Antoinette et du petitfils Emmanuel, qui apparaissent au début et à la fin du roman de MarieClaire Blais, sont la parfaite illustration. Les rapports entre les deux personnages, et le sens que l'un et l'autre manifestent dans l'économie générale de l'œuvre, ne peuvent se découvrir dans le schéma historique de la succession péremptoire. Ni Emmanuel n'est le successeur, le porteur d'avenir, qui révoquerait le monde ancien de Grand-Mère Antoinette; ni celle-ci ne représente un monde ancien qui aurait tout pouvoir sur le nouveau, et le forcerait à la répétition. Ils existent dans et par un roman qui, précisément, récuse ce type d'opposition. Ce qui frappe d'abord, dans Une saison, c'est que Marie-Claire Blais semble citer le roman de la terre, et plus généralement les textes innombrables qui, au Canada français, ont célébré l'idéologie terrienne. Aucun thème, en effet, aucun personnage-type, ne manque à l'appel: le père esclave de la terre et maître de la famille, la mère définie par une abondante procréation; I'influence du curé; la vocation religieuse (Héloïse, Jean-Le Maigre); le messianisme, le mythe de la race supérieure, évoqué à plusieurs reprises par Jean-Le Maigre; liée à ce mythe, la nécessité de l'instruction, de la possession des valeurs intellectuelles et morales. II va sans dire que cette thématique est profondément, radicalement bouleversée par Marie-Claire Blais. Mais de quelle sorte de bouleversement s'agit-il? On a parlé trop facilement de "dénonciation", de "liquidation", ou encore - je cite Goldmann - du "mépris" de de la "haine" de la romancière pour "la société traditionnelle " ${ }^{8}$, com- 
me si, en entrant dans le roman de Marie-Claire Blais, les thèmes du roman de la terre passaient tout simplement du positif au négatif. II y a bien rabaissement, mais sur le mode de ce que Mikkaill Bakhtine, dans son Rabelais, appelle "le réalisme grotesque". (Faut-il souligner qu'il y a du comique dans Une saison?) “Rabaisser, dit Bakhtine, consiste à rapprocher de la terre, à communier avec la terre comprise comme un principe d'absorption en même temps que de naissance: en rabaissant, on ensevelit et on sème du même coup, on donne la mort pour redonner le jour ensuite, mieux et plus. Rabaisser, cela veut dire faire communier avec la vie de la partie inférieure du corps, celle du ventre et des organes génitaux, par conséquent avec des actes comme l'accouplement, la conception, la grossesse, l'accouchement, l'absorption de nourriture, la satisfaction des besoins naturels." 9 Cette prédominance du "bas", du principe matériel ou corporel, est partout visible dans Une saison; les plus hautes fonctions, les opérations les plus nobles, y sont constamment ramenées. Les poèmes de Jean-Le Maigre sont cachés sous les planches des latrines, et l'on sait quelle forme, la nuit, prend pour lui l'amour fraternel. Héloïse, la mystique Héloïse, passe tout naturellement du couvent au bordel; sa vocation s'épanouit, pour ainsi dire, corporellement. Et tout ce monde pense mangeaille, estomac: le père, Héloïse, Jean-Le Maigre, l'oncle Armandin, pour qui les gâteaux de sa femme sont le sens même de la vie. Le récit par Jean-Le Maigre, de sa propre naissance - qu'on a d'ailleurs rapproché du récit de la naissance de Pantagruel ${ }^{10}$-, est l'apothéose de l'absorption par la terre et de la résurrection. «Pivoine retourna à la terre sans se plaindre et moi j'en sortis en criant. Mais non seulement je criais, mais ma mère criait elle aussi de douleur, et pour recouvrir nos cris, mon père égorgeait joyeusement un cochon dans l'étable! Quelle journée! Le sang coulait en abondance... ${ }^{11}$ Cette image, et beaucoup d'autres, qui brouillent la distinction entre la forme humaine et la forme animale, ne sont pas dépréciatives, comme le pense Madeleine Greffard ${ }^{12}$, mais au contraire elles impliquent une revitalisation, une remise en mouvement de thèmes et de personnages figés par l'habitude.

Cette remise en mouvement, en circulation ( 'Le sang coulait en abondance..."), cette mobilité et cet inachèvement des formes, qui sont des effets du grotesque, entraînent la perte du pouvoir pour ceux qui le détenaient dans l'idéologie terrienne - car le pouvoir appartient à l'ordre de l'achevé, du stable. Voici le père, figure traditionnelle de la maîtrise: "La voix d'homme n'est qu'un murmure. Elle se perd, disparaît. "13 Portrait de la mère: “Debout contre le mur, la tête un peu renversée sur l'épaule, sa mère écoute en silence. Elle dort peut-être. Sa robe est ouverte sur un sein pâle qui fléchit. " ${ }^{14}$ Le curé s'en tire un peu mieux, mais on reconnaîtra difficilement dans ce personnage mou, tout à ses aises, grand mangeur, buveur de bière même en carême, la forte image autoritaire du pasteur d'autrefois. Ces personnages, point n'est besoin de les contester, de les dénoncer, de les liquider; ils se dissolvent d'eux-mêmes, dans un monde en- 
tièrement gouverné par le «principe matériel», le mouvement infini de la mort et de la régénération.

Nous revenons à Grand-Mère Antoinette et nous découvrons que, seule parmi les représentants de l'ordre ancien, elle conserve un pouvoir. Qu'elle appartienne à cet ordre, impossible d'en douter; elle n'est pas une grand-mère à la mode. "Moi aussi, dit-elle, je suis contre le progrès." 15 Elle est soumise au curé, comme elle fut soumise, en rechignant, à son défunt mari. Son pouvoir est donc celui de l'ordre ancien, mais distendu, élargi aux dimensions du cosmos; elle est «immense", sa taille est un "antre" sous des «montagnes de linge»; elle est "un fleuve chaud"; et elle a le temps, tout le temps: "Nous aurons beaucoup de temps, dit Grand-Mère, rien ne presse pour aujourd'hui... "Enfin, GrandMère Antoinette est la maîtresse - et, en cela, son pouvoir diffère radicalement de celui que pourraient détenir le père et la mère - du paradoxe, du mouvement par lequel, depuis toujours, la terre absorbe et rend la vie. C'est en elle et par elle que meurt et vit Jean-Le Maigre. "Une bien triste mort en vérité. Mais elle n'en croyait rien. Jamais Jean-Le Maigre ne lui avait paru aussi vertueux que depuis l'heure de sa mort, jamais il ne lui avait paru en aussi bonne santé que depuis qu'il était dans sa tombe bien tranquille, là-haut, sur la colline "... ${ }^{17}$ C'est d'elle également que naît véritablement Emmanuel. En Grand-Mère Antoinette, le monde ancien ne s'oppose pas au nouveau car elle incarne, selon la formule de Bakhtine, "l'éternel inachèvement de l'existence $» 18$.

Chez Jacques Ferron, le pari sur l'ancien paraît beaucoup plus net que chez Marie-Claire Blais. C'est que Ferron, en trois pages ou en cinq cent, ne pratique jamais que la forme du conte, et que le conte a partie liée avec l'ancien, par l'usage qu'il fait du merveilleux et de la sagesse des nations. Comparons, par exemple, le curé d'Une saison au chanoine Godfrey des Contes anglais. Le premier, comme je l'ai déjà montré, est un bonhomme assez sympathique, inoffensif, dont ni les paroles ni les actes ne prêtent à conséquence: un tigre à qui l'on aurait enlevé les dents et les griffes. Au premier abord, le chanoine Godfrey ne semble pas imposer une image beaucoup plus forte. Le voici qui arrive au chevet de la Dame de Ferme-Neuve, où se trouve déjà le médecin-narrateur. «ll ôta son manteau, ne me demanda même pas si elle était morte; il attaqua d'emblée ses conjurations. Je m'agenouillai. Il avait du métier ce curé, et même une sorte de talent naturel: gueuler du nez est beaucoup plus efficace que de la bouche. C'est péremptoire, ça n'accroche jamais les sanglots, cela vous donne un brouhaha d'oiseaú qui couvre tout et réduit la peine de la famille à quelques pauvres petits reniflements. "19 II est assez évident que le narrateur se gausse du chanoine, et qu'il ne croit pas à l'efficacité de ses "conjurations". Mais, assimilant par ce mot le chanoine à quelque sorcier de village, Ferron le fait entrer dans le monde du merveilleux qui est, pour lui, le monde de la signifiance. Comme Monseigneur Camille Roy et tous les curés de Jacques Ferron, le chanoine Godfrey est I'homme de la cérémonie, du rite, de la bonne compagnie. Et la cérémonie - même privée de 
pouvoir; et peut-être parce qu'elle est dissociée du pouvoir - n'est pas une vaine affaire, puisque par elle, par le langage qui est lui-même cérémonie, se rétablit l'accord sans cesse menacé de l'homme et du monde.

La cérémonie est, par définition, ancienne; elle ne vaut que mille fois répétée. Elle fleurit naturellement au village ${ }^{20}$, à la campagne, et survit difficilement dans l'appétit de nouveau, de changement perpétuel, que propose la ville. Le conteur, donc, ne peut que refuser la ville - sauf quand parfois, la nuit, elle s'ouvre aux sortilèges. Quittant la rive sud il lui arrive de traverser le pont Jacques-Cartier pour se rendre à Montréal, changeant "de monde en changeant de rive", "tout à (s)a hâte de rencontres" dont il attend beaucoup. Mais le pont ne devient beau, riche de sens, que lorsque le narrateur revient, "fatigué, déçu». "La nuit, dit-il, le fermait comme une cathédrale. Je m'y engageais avec remords, regrettant le temps perdu et les devoirs oubliés. La haute voûte noire finissait en s'ouvrant sur les feux des plus basses étoiles et les lumières de la plaine qui s'étend de Longueuil à Chambly, de Saint-Lambert à Saint-Amable, feux et lumières diversement groupés selon les constellations, les petits villages et les grands faubourgs. Les monts Saint-Bruno, Beloeil et Rougemont dressaient de sombres massits. au milieu de ce scintillement. "21 Ce qui scintille, ce qui brille ainsi, dans la direction opposée à celle de la ville, c'est la lumière qui vient $\mathrm{du}$ fond des temps, du fond de l'histoire - et qui. dans l'ordre textuel, est signalé par la forme même du conte, par le retour obligé dans le conte des plus anciens motifs de la figuration humaine.

Cependant, ce retour à l'ancien n'a rien de passéiste, et Novalis a pu dire que "le conteur authentique est un visionnaire du futur" ${ }^{22}$. Car si, pour le romancier réaliste, attaché au temps linéaire de l'histoire, l'idéologie terrienne représente ce qui est figé, mort, ce qui doit être quitté pour la mouvance et le risque de l'aventure urbaine, il en va tout autrement pour le conteur, qui habite le temps circulaire du mythe; pour celui-ci, la ville est l'univers du donné, de l'achevé, alors que la campagne (l'ancien) est l'image du possible. Ferron le sait bien, que le village, le TrompeSouris, est devenu "inaccessible» ${ }^{23}$, et qu'on ne met pas "une pièce de drap neuf à un vieux vêtement (...), du vin nouveau dans de vieilles outres" ${ }^{24}$. Entre la ville et la campagne, entre le donné et le possible, il rêve d'un équilibre, ou mieux, d'un "échange». Il parle ainsi du vagabond, dans "Suite à Martine": "La campagne entrait dans la ville pendant que le vagabond, son sac sur le dos, en sortait. II y avait échange d'esprit et de santé entre la ville et la campagne. Les échanges ont cessé. La ville a gardé son bien, la campagne le sien, avec le résultat qu'elles ont tout perdu et l'esprit et la santé. L'un ne va pas sans l'autre. II y a des échanges nécessaires. 25 De ces échanges, Jacques Ferron parle au passé, comme s'ils étaient révolus; mais que fait son œuvre," son écriture, sinon renouer toujours ces liens rompus, faire ressurgir dans le présent urbain, pour l'ouvrir au possible, la provocation du merveilleux? Dans cette perspective, l'ancien n'est pas un état, une chose, une substance, qui voudrait supplanter le nouveau; il est, à l'écart de toute notion chronologique, l'autre du 
nouveau, ce qui le tire de sa suffisance, de son achèvement, pour le relancer sur la voie de l'avenir.

Pour passer chez Réjean Ducharme c'est le clergé, encore, qui nous servira de transition. Nous avons donc rencontré, chez Marie-Claire Blais, un curé bonhomme, grand buveur de bière; chez Jacques Ferron, un Monseigneur de qualité et un chanoine habile en conjurations; il nous manquait un vicaire. Le voici: «Je parle, dit Réjean Ducharme, comme un vicaire. ${ }^{26}$ Non pas un vicaire de gauche, dans le vent, animateur de comités de citoyens; mais un vicaire tout ce qu'il y a de plus vicaire, vitupérant la ville et ses dangers, le syndicalisme et ses excès, la chair et ses turpitudes, l'anti-moderne par excellence. L'avant-garde, pour lui, c'est la grande illusion: «Comme tous les êtres humains fuient, dit Ducharme, ceux qui sont à l'avant-garde sont ceux qui fuient le plus vite. ${ }^{27}$ La solution s'impose d'elle-même: "Restons en arrière, avec Crémazie, avec Marie-Victorin, avec Marie de l'Incarnation, avec lberville et ses frères héroïques. Restons en arrière. Restons où nous sommes. N'avançons pas d'un seul pas. Restons fidèles. Souvenons-nous. " ${ }^{28}$ Monseigneur Camille Roy n'aurait pas dit mieux que ce vicaire à la langue bien pendue: il faut retourner au Régime français et aux mœurs pures de nos ancêtres.

Mais notre vicaire, comme de bien entendu, a le nez qui voque. II est engagé dans ce que Michel Van Schendel a appelé un «système d'outrance "29, qui fait que chacune de ses affirmations s'abolit, s'annule, dans l'excès même qui lui donne son éclat. Si nous le suivons dans sa marche arrière, son mouvement rétrograde, nous arrivons très vite au suicide - celui de Chateaugué par exemple. Nous arrivons au “bérénicien", qui est une. langue très pure, échappant à la pollution de l'usage, mais dont le coefficient de communication n'est pas très élevé. Nous arrivons à úne enfance si pure, si libre, si blanche, qu'en toute vraisemblance elle n'a jamais existé. Pas plus que Jacques Ferron ne croit à la viabilité du village de TrompeSouris, Réjean Ducharme ne croit que les espaces vierges dont rêve Mille Milles, Labrador et Ungava, ou encore le Port-Burwell où Chateaugué aurait reçu le nom d'Ivugivic, soient des lieux, des états, qu'on pourrait préserver, auxquels on pourrait retourner. Parce qu'il tient son journal intime, parce qu'il est du côté de l'écriture, du roman - et tout romancier est réaliste, veut veut pas - Mille Milles doit mettre en doute les origines de Chateaugué. "Tu n'es pas une vraie Esquimaude, lui dit-il. Tu es blonde, tu as le visage étroit et tu n'as pas les yeux en amande." Chateaugué n'en est pas moins, sous un autre rapport, Esquimaude, et plus profondément Esquimaude que toutes celles qui ont les yeux en amande et les cheveux noirs. Car, ainsi que le disait Ssouvie à Asie Azothe : "Il n'y a que ce que tu inventes, que ce que tu crées. " ${ }^{31}$

Attachons-nous à cette idée d'invention; et examinons-la dans ses rapports avec le langage, avec l'écriture, puisque l'essentiel, chez Ducharme, se joue là. Est-il besoin de démontrer que la densité visible du lu et de l'écrit, dans l'œuvre de Ducharme, est particulièrement forte? Que la ré- 
férence aux cuvres (de la Chanson de Roland à Lautréamont en passant par Nelligan et Rimbaud), aux genres littéraires (roman, chanson de geste, épopée), au fonctionnement de la langue (grammaire, figures de rhétorique), est, dans ses romans, plus riche de sens que la référence au vécu? Il s'agit donc d'inventer; de produire du nouveau, de l'original, de l'originel. Mais il existe deux façons de concevoir l'originel, et qui commandent des opérations différentes. La première est fondée sur le refus de l'ancien, de l'héritage, sur la revendication passionnée de l'autonomie personnelle. Cette revendication ne peut qu'être agressive, parce qu'elle répond à l'agression de la culture, de l'écriture reçues. "Quand j'ai quitté l'école, dit Mille Milles, j'étais plein de noms comme on est plein de scarlatine.»32 Inventer une écriture, ce sera dresser l'agression personnelle contre l'agression commune, ainsi qu'on le voit par l'exemple de Bérénice Einberg. Ce sera aussi, à la limite, épuiser cette double agression, cette double violence, et c'est ce qui se passe dans L'avalée des avalés où Bérénice, en tuant Gloria - Gloria, la guerre, l'agression, la violence -, s'élimine elle-même comme contre-violence. Ainsi finit le personnage, ainsi finit le roman. Combattre la culture reçue, c'est participer de cette culture, puisqu'elle se définit par la violence, le combat; et toute victoire sur l'héritage culturel ne peut qu'entraîner la mort du sujet.

Mais l'originel, dans l'œeuvre de Réjean Ducharme, prend également un autre sens, défini par les mots d'âme, de pureté, d'amitié; par les noms de Jeanne d'Arc, de Nelligan, de Rimbaud (et de sa sœur Isabelle), de Marie de l'Incarnation, par la Flore laurentienne du Frère Marie-Victorin; par des paysages blancs, Alaska, Ungava, Nouveau-Québec, ou des époques anciennes, comme le Régime français du Canada. Ces mots ne désignent pas un héritage. On n'hérite pas de Nelligan, de Rimbaud, de l'Alaska ou du Régime français. Au contraire, ils représentent ce qui est perdu, et doit le demeurer; ce qui est passé, radicalement, "du dépassé, du trépassé, du déclassé, du crétacé, du miel à mouches, de la rhubarbe à cochons ". Au passé de la culture reçue, encore active, qui nourrit le langage de l'agression, s'oppose le passé du non-pouvoir, le passé des lointains de l'âme, source d'un autre langage, celui de Asie Azothe, de Colombe Colomb, de Constance Chlore, de Chateaugué : "Avec elle, il faut que tout ce qu'on dit ne veuille rien dire. II faut la laisser être. " ${ }^{34}$ Si Réjean Ducharme manifeste une telle prédilection pour les poèmes de Nelligan et la Flore laurentienne, c'est que voilà deux œuvres où l'imposition du dire s'efface, laisse toute la place à la présence, à l'amitié, celle de l'âme ou celle de la plante, celle d'un texte sans leçon. Ainsi se forme un lien entre l'original (le nouveau) et l'originel (l'ancien) qui déborde assurément les perspectives de notre vicaire, si bien embouché qu'il soit.

II semble assez évident, d'après les brèves analyses auxquelles je me suis livré, que les rapports entre l'ancien et le nouveau, dans les œuvres de Réjean Ducharme, Marie-Claire Blais, et Jacques Ferron, sont établis par des manœuvres scripturaires très différentes les unes des autres; mais, aussi bien, qu'ils aboutissent à une loi commune. On remarque d'abord que le 
nouveau - c'est-à-dire l'cuvre elle-même, en tant qu'elle est innovation récuse le passé immédiat, l'héritage direct : chez Marie-Claire Blais, les parents se dissolvent dans l'insignifiance; dans les contes et romans de Ferron, les signes de la tradition récente, Saint-Denys-Garneau, Montréal ou même le Trompe-Souris, sont frappés de caducité; dans l'œuvre de Ducharme, enfin, c'est tout le langage de la rationalité moderne, tel qu'il s'est développé depuis un siècle ou deux, qui est agressé, neutralisé. Le nouveau, donc, brise avec l'ancien. Mais en revanche il renoue avec le plus ancien: Grand-Mère Antoinette, Marie de I'Incarnation et le dieu amérindien Isou Manichou auquel Jacques Ferron donne la parole dans Le ciel de Québec. Marshall McLuhan a décrit cette double opération dans son dernier ouvrage, Du cliché à l'archétype : «...L'introduction d'une nouvelle technologie, écrit-il, a pour effet de transformer celle qui la précède en objet de rebut, qui est ainsi libéré de son ordre fonctionnel pour être immédiatement réorienté sous forme de cliché-sonde par l'artiste. L'invention de Gutenberg expulse le Moyen-Âge et récupère l'Antiquité gréco-latine. Aujourd'hui, les systèmes et les encodeurs électroniques ont "périmé " la ferraille et la mécanique industrielle et récupéré toute la collection de cultures archaïques et prélittéraires. " ${ }^{35}$ L'œuvre de Ducharme, par exemple, que nous pouvons assimiler à une nouvelle technologie, transforme le discours du vicaire en "un objet de rebut", c'est-à-dire qu'elle le retire du circuit de l'usage, du pouvoir, pour en faire un "cliché-sonde" qui ira "récupérer" les archétypes de Marie de I'Incarnation et de Jeanne d'Arc. De la même façon, Jacques Ferron "périme" le chanoine Godfrey, et Marie-Claire Blais I'image parentale. L'archétype cependant n'est pas ici, comme dans la pensée de Jung, un substrat élémentaire permanent de l'expérience humaine. II se propose, non pas comme substance, chose, mais comme rapport, comme jeu de rapports ; “jeu " de l'intervalle, dit McLuhan, et non pas de la connexion " ${ }^{36}$. C'est la distance même, et la distance maintenue, qui est garante de la fécondité et de l'actualité du rapport. Les récits de Blais, Ferron et Ducharme sont vraiment de notre temps, de notre monde, en ce qu'ils disent la distance, le rapport, l'intervalle - l' «échange», dit Ferron -, plutôt que les choses mêmes dans l'ordre linéaire de la causalité.

Ne disent-ils pas, également, la fin du roman? Je parle du roman classique, selon la définition implicite qu'en ont donné au $19^{\mathrm{e}}$ siècle un grand nombre d'œuvres prestigieuses, le roman du passé simple et de la troisième personne, dont Roland Barthes a montré qu'il était formellement apparenté au récit historique déroulant la succession des causes et des effets ${ }^{37}$. Ce roman n'a régné que durant peu d'années au Québec, avant et après la Seconde Guerre Mondiale, et il semble parfois que nous en attendions encore la renaissance, c'est-à-dire des œuvres qui feraient le portrait, aussi ressemblant que possible, de ce que nous sommes. II arrive même que l'on tente de lire les œuvres récentes dans cette optique - ce qui conduit à des contradictions gênantes, comme on l'a vu pour le personnage de Grand-Mère Antoinette. Dans d'autres pays, qui ont profondément vécu l'expérience du $19^{\circ}$ siècle, le roman classique peut se survivre dans une 
certaine mesure, avec quelques modifications. Mais, pour nous, le siècle de la rationalité, de l'histoire, du roman, n'a été qu'une brève parenthèse, tardivement vécue d'ailleurs, et nous sommes entrés sans coup férir, presque sans bagages, dans le nouvel âge, qui est l'âge de l'éclatement. “Un monde qui se fait sauter lui-même, a dit Hermann Broch, ne permet plus qu'on en fasse le portrait " ${ }^{38}$. Notre roman nous représente, représente notre monde, précisément parce qu'il n'a plus de lieu fixe, parce qu'il n'est pas une forme stable. S'il évoque le texte ancien, ce n'est pas par simple piété, comme le faisaient les romans historiques du $19^{\mathrm{e}}$ siècle, pour donner une vie nouvelle aux mythes de l'origine, mais bien pour fabriquer l'image de ce qui se passe, ici et maintenant. Le roman manœuvre comme nous manœuvrons nous-mêmes depuis que l'univers, sous le regard ironique du spoutnik et dans l'œil globuleux de la télévision, est devenu un champ d'expériences où les rapports les moins attendus se font comme d'eux-mêmes.

(janvier 1975)

1. Lucien Goldmann, "Note sur deux romans de Marie-Claire Blais ", dans Structures mentales et création culturelle, Paris, 10/18, p. 359.

2. Madeleine Greffard, "Une saison dans la vie d'Emmanuel, kaléidoscope de la réalité québécoise", dans Littérature canadienne, Cahiers de Sainte-Marie, Montréal, 1966, p. 19.

3. Id., p. 17.

4. Réjean Ducharme, Le nez qui voque, Gallimard, Paris, 1967, p. 49.

5. Réjean Ducharme, La fille de Christophe Colomb, Gallimard, Paris, 1969, p. 196.

6. Jack Warwick, “Un retour aux mythes de la terre?", dans Études françaises, Montréal, PUM, novembre 1973, p. 301.

7. Marthe Robert, L'ancien et le nouveau de Don Quichotte a Kafka, Petite Bibliothèque Payot, Paris, p. 32.

8. Lucien Goldmann, op. cit., p. 362.

9. Mikkaïl Bakhtine, L'xuvre de François Rabelais et la culture populaire au Moyen Âge et sous la Renaissance, trad. Andrée Robel, Gallimard, Paris, Bibliothèque des idées, p. 30.

10. J. Bersani, M. Autrand, J. Lecarme, B. Vercier, La littérature en France depuis 1945, Bordas, Paris, p. 664.

11. Marie-Claire Blais, Une saison dans la vie d'Emmanuel, Montréal, Éditions du Jour, 1965, p. 50.

12. Madeleine Greffard, op. cit., p. 20 : «La dénonciation de la vie se fait de façon puissante au niveau des images. Toutes sont dépréciatives; la majorité ravalent la vie humaine au niveau de la vie animale, insistent sur son état larvaire et absurde.,

13. Marie-Claire Blais, op. cit., p. 11.

14. Id., p. 11-12.

15. Id., p. 103.

16. Id., p. 8.

17. Id., p. 92.

18. Mikkaïl Bakhtine, op. cit., p. 41. 
19. Jacques Ferron, Contes, édition intégrale, Montréal 1968, HMH, p. 185.

20. Voir le plaidoyer de Chubby Power pour les petits villages dans Le ciel de Québec, Montréal, Éditions du Jour, 1969, p. 99-100.

21. Contes, p. 48.

22. Cité par André Jolles, Formes simples, trad. Antoine Marie Buguet, Paris, Éditions du Seuil, collection Poétique, p. 182.

23. Contes, p. 98.

24. Id., p. 131.

25. Id., p. 127.

26. Réjean Ducharme, Le nez qui voque, p. 50.

27. Id., p. 66.

28. Id., p. 29.

29. Michel Van Schendel, "Ducharme l'inquiétant", dans Littérature canadiennefrançaise, Conférences J.-A. de Sève, Montréal, PUM, 1969, p. 232.

30. Le nez qui voque, p. 87.

31. Réjean Ducharme, L'océantume, Paris, Gallimard, 1968, p. 112.

32. Le nez qui voque, p. 104.

33. Id., p. 79.

34. Id., p. 56.

35. Marshall McLuhan avec Wilfred Watson, Du cliché à l'archétype, la foire du sens, trad. Derrick de Kerckhove, Montréal, Hurtubise-HMH, p. 102.

36. Id., p. 163.

37. Roland Barthes, Le degré zéro de l'écriture, Paris, Médiations, p. 29 sequ.

38. Hermann Broch, Création littéraire et connaissance, trad. Albert Kohn, Paris, Gallimard, Bibliothèque des idées, p. 255. 